\title{
Multi-channel phasemeter and its application in the heterodyne laser interferometry
}

\author{
LIU HeShan ${ }^{1,2 \dagger}$, DONG YuHui ${ }^{1,2 \dagger}$, LUO ZiRen $^{1}$, LI YuQiong $^{1} \&$ JIN Gang ${ }^{1 *}$ \\ ${ }^{1}$ National Microgravity Laboratory (NML), Institute of Mechanics, Chinese Academy of Sciences, Beijing 100190, China; \\ ${ }^{2}$ School of Physics, Chinese Academy of Sciences, Beijing 100190, China
}

Received October 8, 2014; accepted December 23, 2014; published online January 29, 2015

\begin{abstract}
The mission to detect gravitational wave in space requires a not only sophisticated but also ultra-precise laser interferometric measurement system. Within a single spacecraft, tens of interferometric beat signals are generated at the same time and they also need to be processed simultaneously. In this paper, a multi-channel phasemeter which can parallelly process the signals is constructed. The test shows that a sensitivity of $2 \pi \mu \mathrm{rad} / \sqrt{ } \mathrm{Hz}$ could be achieved in the frequency range of $0.1 \mathrm{Ho} 10 \mathrm{~Hz}$. We also utilize the phasemeter to evaluate the performance of a heterodyne laser interferometer.
\end{abstract}

phasemeter, laser interferometer, gravitational waves detection

Citation: Liu H S, Dong Y H, Luo Z R, et al. Multi-channel phasemeter and its application in the heterodyne laser interferometry. Sci China Tech Sci, 2015, 58: 746-749, doi: 10.1007/s11431-015-5770-y

\section{Introduction}

Nowadays, detection of gravitational wave (G.W.) in space has attracted much attention in the scientific community $[1,2]$. Many proposals, such as laser interferometer space antenna (LISA), evolved laser interferometer space antenna (eLISA), big bang observer (BBO), DECi-hertz interferometer gravitational wave observatory (DECIGO) and super astrodynamical space test of relativity using optical devices (ASTROD), are put forward and taken into research [1-5]. When a train of G.W. passes by, it induces phase change within the carrier laser [6]. Due to the weakness of G.W., the phase change can be as small as $2 \pi \mu \mathrm{rad} / \sqrt{\mathrm{Hz}}$ at frequencies between $0.1 \mathrm{mHz}$ to $1 \mathrm{~Hz}$ [7-10]. For this reason, there are two problems we have to confront with. Firstly, how can we measure such small phase change in the frequency band as mentioned before? Secondly, how can we

*Corresponding author (email: gajin@imech.ac.cn)

$\dagger$ Contributed equally to this work. precisely calibrate the phase noises which come from an interferometer, optical component, transmitting wire, photo detector, etc.? A digital phasemeter, which is built upon field programmable gate array (FPGA) and based on digital phase-locked loop (DPLL) scheme, is thought to be the best solution to the above problems [11-14]. Such a phasemeter with phase sensitivity of $2 \pi \mu \mathrm{rad} / \sqrt{\mathrm{Hz}}$ in the frequency range of $1 \mathrm{mHz}$ to $1 \mathrm{~Hz}$ has been built by the Albert Einstein Institute (AEI, Germany) and the Jet Propulsion Laboratory (JPL, America) [9,15-18].

The Chinese gravitational waves detection mission is on the agenda, and the verification of the related technologies is also under way [19]. In our previous work, a prototype of phasemeter for the principle demonstration was built up and evaluated [20]. However, the prototype is only a singlechannel phasemeter which also needs a reference signal to demodulate the phase from the carrier input signal. In G.W. detection, there are more than 20 signals in one spacecraft, and all the signals need to be processed in a parallel way [21-24]. In this paper, an upgraded multi-channel phaseme- 
ter, which is compatible with a multi-processing environment, is constructed. It also offers a highly precise way to calibrate the phase noise which comes from an interferometer, optical component, transmitting wire, photo detector, etc. Then, the architecture of the multi-channel phasemeter is described in detail. After that, the phasemeter is tested with a signal generator, and the possible noise sources are also discussed. At last, the phasemeter is utilized to evaluate the performance of a laser interferometer system.

\section{Architecture and design}

The architecture of the multi-channel phasemeter is shown in Figure 1. The phasemeter is implemented on a commercial FPGA board (DE3-340, Terasic), and the program is compiled by the verilog hardware description language (Verilog HDL). The phasemeter mainly includes two analog to digital converter (ADC) (AD9254, Analog Devices) boards and a FPGA board in hardware. The detected signals are modulated and digitized by the ADC board, and then enter into the FPGA for the DPLL algorithm realization. The ADC board firstly needs to be aroused by relevant driver program. The digitized signals are respectively multiplied by a numerically controlled oscillator (NCO) with sine and cosine signals in the same frequency. The mixed signals are filtered by low pass filters (LPFs) to remove the multiplied frequency parts. The remained value after filtering is proportional to the related phase difference between the detected signal and the NCO. In order to simplifying the feedback part, a Nios II processor is constructed in the FPGA. Before being entered into the processor, data type should be translated from the integer to the float by the data type convertor (DTC) parts. The processor accomplishes the arithmetic of phase calculation, frequency feedback and the communication with computer. The proportional integral (PI) controller, which is the frequency feedback part, will make the frequency of the NCO follow with the detected signal. Finally, four series information can be chosen for further analysis. To decrease the phase noise caused by the integration of frequency error [9], the multi-channel phasemeter introduces one feedback loop, and the frequencies of NCOs between channels are changed synchronously. So, the multi-channel phasemeter is mainly used in the ground interferometer demonstration, in which the frequency fluctuation between interferometer arms can be neglected. In the phasemeter system, all the parts are synchronized by an oscillator. Figure 2 shows the physical picture of the multi-channel phasemeter.

\section{Experiments and discussion}

Before calibrating an interferometer or other devices, the multi-channel phasemeter firstly needs to be verified. The experiment is implemented under the condition that the tested signal is generated by a functional generator (33522A, Agilent). The frequency of the tested signal is set at $1 \mathrm{MHz}$. To reduce the influence of phase error between different channels, one signal from the functional generator is split into four and connects with four channels of the phasemeter respectively. The response uniformity between channels is our concerned problem in the experiment. The results of the test are shown in Figure 3.

From the results of Figure 3, all the curves show flat except for a few peaks and reach the level of $2 \pi \mu \mathrm{rad} / \sqrt{\mathrm{Hz}}$ in the frequency of 0.1 and $10 \mathrm{~Hz}$. The noise level is dominated by the electronic noise of the tested signal. Furthermore, it is noticed that some peaks also appear in the frequency range, and only the blue and the red curves have the spikes. The peaks, which appear in the second and higher harmonics of the initial frequency difference between the detected signal and the NCO, are the remained noise of the feedback
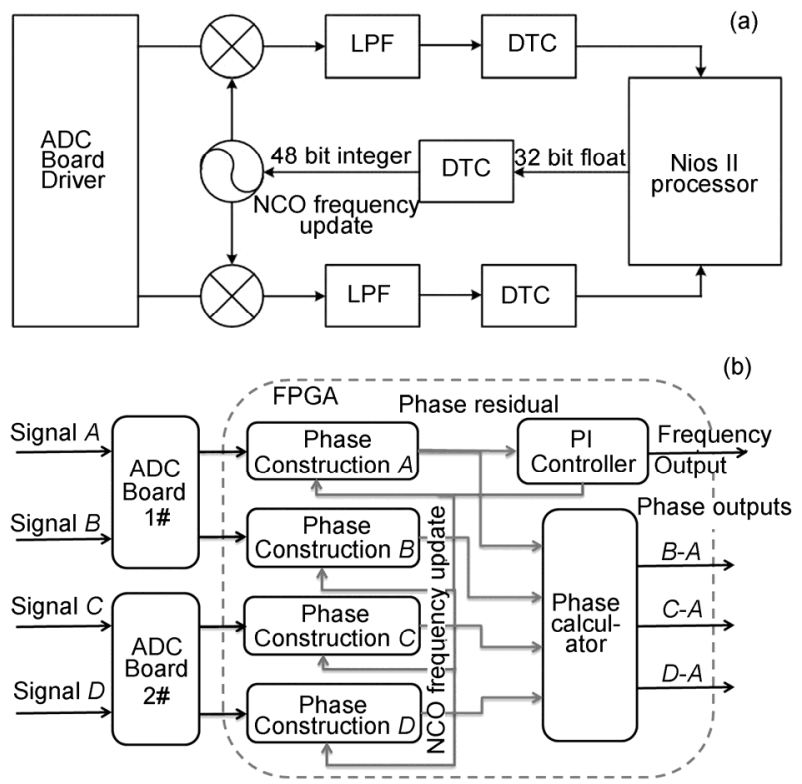

Figure 1 Architecture of the phasemeter, which is implemented on a FPGA platform. (a) The detailed architecture of one channel; (b) the whole architecture of the multi-channel phasemeter.

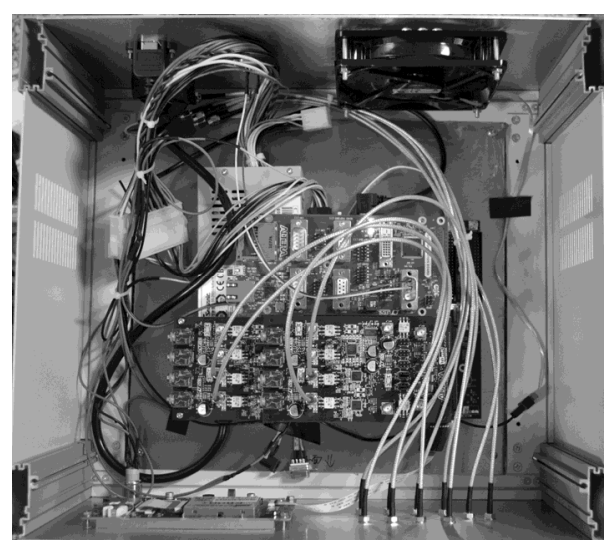

Figure 2 Physical picture of the multi-channel phasemeter. 


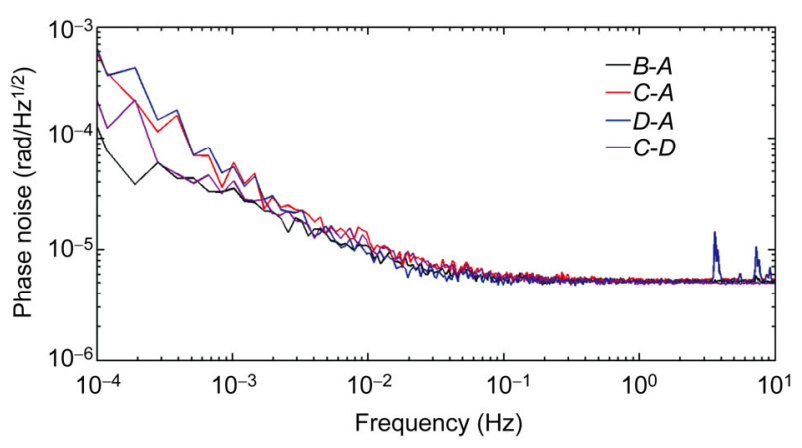

Figure 3 (Color online) The experiment results are obtained in the condition that the tested signal is generated by a functional generator. The results have been smoothed by the method of linear amplitude spectrum density (LASD).

part. The detected signals are the same for each channel of the phasemeter, so the peaks are expected to appear in the results of every channel. Actually, the peaks can be removed through the subtraction in principle, for the output of the phasemeter is the phase difference between channels. However, the output value is not the desired phase difference since different channels have different time delays in the algorithm processing. Therefore, the value of time difference between the channels determines the peaks value [20]. All the algorithms after DTC are carried out in the Nios II processor whose architecture is a serial mode. So, the phase information of different channels is successively gained in the Nios II processor. Although the time difference is short enough between the channels of phasemeter, the effect still appears in the results of non-adjacent channels. In the following research, multiple Nios II or DSP hardware processors will be introduced to reduce the noise. It also can be seen from Figure 3 that the noise floor of different curves increases to different degrees in the frequency below $0.1 \mathrm{~Hz}$. In our previous research, the noise was mainly caused by the thermal drift, and the temperature fluctuation mainly influenced the analog frontend of the ADC board [20]. In the multi-channel phasemeter, there are two cooling fans for the thermal dissipation which may aggravate the temperature fluctuation. Besides, limited by the hardware condition, four ADCs and their analog frontends are distributed in two ADC boards which may lead the temperature of the frontends especially in different boards to largely different.

From the above analysis, the response of the phasemeter shows discordances among the channels in different degrees. The discordances result from the software level as well as the hardware limit. In the next stage, customized board and thermal insulation methods will be adopted to decrease the effect. The Verilog HDL code of the phasemeter also needs to be optimized.

In the above test, the phasemeter has been proved to function well. However, some problems have also arisen. Next, the phasemeter will be used to evaluate a simple in- terferometer, which is a part of our measurement system. The schematic diagram of the interferometer, which is implemented on an aluminum alloy optical bench, is shown in Figure 4. The laser beam stimulated by a laser is split into two beams by a $5 / 5$ beam-splitter mirror. One of the beams directly accesses to an AOM, while the other beam accesses to another AOM after its spread direction is changed by a reflector. Both of the beams have different modulation frequencies values when they go through the AOMs. The difference between the two modulation values exists in the frequency of the heterodyne signal. After several functional units, an interfered signal is formed, and detected by PDs. The heterodyne frequency is also set at $1 \mathrm{MHz}$. The common mode noise especially the laser frequency jitter can be largely suppressed, for the interferometer is an equal arm design. In order to eliminate the influence of different phasemeter channels, only $A$ and $B$ channels are used here. The results are shown in Figure 5.

From the red curve in Figure 5, the noise curve is flat within the whole frequency range, and the sensitivity only reaches $0.6 \mathrm{mrad} / \sqrt{\mathrm{Hz}}$. The noises, as Figure 3 shown, are submerged in larger noises. Obviously, the noise is dominated by the electronic noise of the interferometer system. Decreasing the electronic noise is the feasible way to improve the sensitivity of the interferometer at present. From

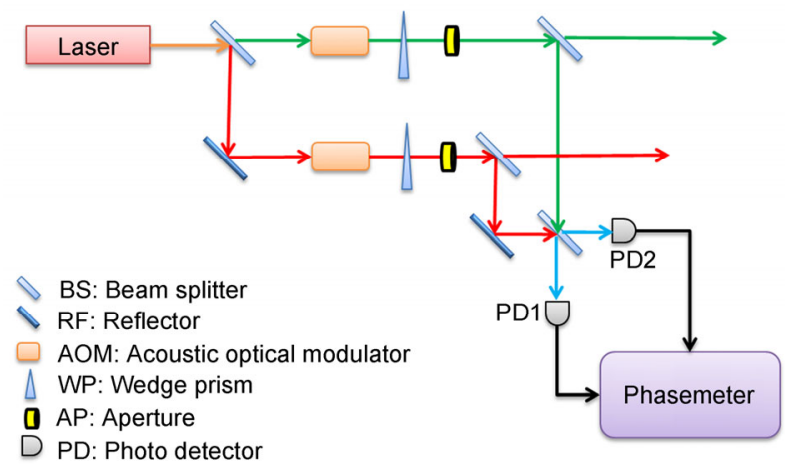

Figure 4 (Color online) Schematic diagram of the interferometer.

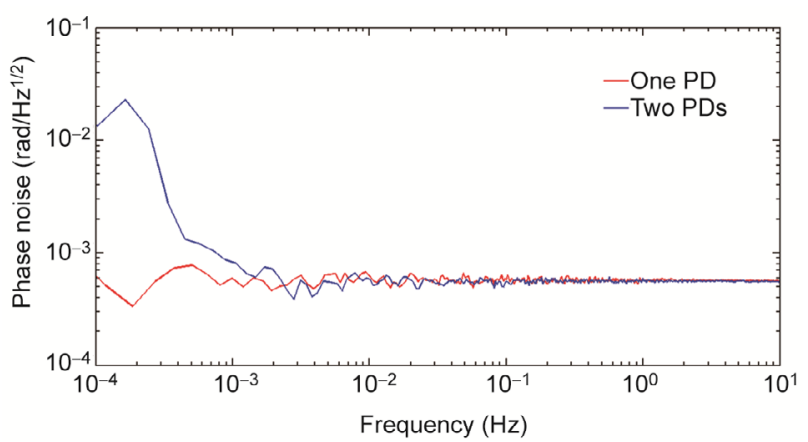

Figure 5 (Color online) The experiment results of the phasemeter are tested by an interferometer, and the data has also been smoothed by the method of LASD. The red curve is the result in the condition that the signals are split by PD1. The blue curve is the result in the condition that the tested signals are generated by PD1 and PD2. 
the blue curve, the signals from the two PDs have a consistent response in the frequencies between $10 \mathrm{~Hz}$ and 1 $\mathrm{mHz}$. However, in the frequency range of $0.1 \mathrm{mHz}$ and 1 $\mathrm{mHz}$, the responses are largely different. The reasons are analyzed in the following: (1) The comparisons of the red curve indicate that one of the noises possibly comes from the large temperature fluctuations. However, both of the tests are implemented under similar environmental condition. It is impossible for the temperature to be distributed so differently. (2) The PDs used in the interferometer has not been calibrated in advance. This may be the reason why the phase responses are greatly different in the frequency between $1 \mathrm{mHz}$ and $0.1 \mathrm{mHz}$. (3) Due to vibration or thermal drift of the environment, the laser beams are vibrated along with the optical bench. It will introduce different wave-front misalignment in the two PDs. The detailed information about the wave-front misalignment can be obtained from the related references about the laser beam pointing control of the G.W. detection mission $[23,24]$. To solve this problem, the best way is to enhance the stability of the optical bench and decrease the thermal drift of the environment.

From the above analysis, several problems are brought out in the present interferometer. Muffling the electronic noise, calibrating the PDs, enhancing the stability of the optical bench and decreasing the thermal drift of the environment are the main tasks to improve the sensitivity of the interferometer.

\section{Conclusions}

Within a single spacecraft, tens of interferometric beat signals are generated at the same time and they also need to be processed simultaneously. A multi-channel phasemeter which can parallelly process the signals is constructed in the paper. The test shows that the sensitivity of the phasemeter could achieve $2 \pi \mu \mathrm{rad} / \sqrt{\mathrm{Hz}}$ in the frequency range of 0.1 $\mathrm{Hz}$ to $10 \mathrm{~Hz}$. Due to the different ADC boards between channels, the thermal noise increases largely in the frequencies below $0.1 \mathrm{~Hz}$. And, some peaks caused by the feedback loop also appear in the non-adjacent channels phase readout. Customized board and thermal insulation methods will be adopted to decrease the effect in future study. The phasemeter is also employed to evaluate the performance of a simple laser interferometer. In the test, we have found that the electronic noise is the main factor for the low sensitivity of the interferometer. Moreover, the signals beside the BS are largely different in the frequency from $1 \mathrm{mHz}$ to 0.1 $\mathrm{mHz}$. These noises may arise from the different phase responses between the two PDs at two output side of BS and the wave-front misalignment caused by the laser beam jitter.

Improving the sensitivity of the interferometer system is a challenging task. We need to find out the major noise source. So, the phase response calibration to the interferom- eter, optical component, transmitting wire, photo detector and etc will be the main tasks in our future investigation.

This work was supported by the Scientific Equipment Development and Research Project of Chinese Academy of Sciences (Grant No. Y231411YB1), and the Space Science Research Projects in Advance of Chinese Academy of Sciences (Grant No. O930143XM1).

1 Pitkin M, Reid S, Rowan S, et al. Gravitational wave detection by interferometry (ground and space). Living Rev Relativity, 2011, 14: 13-20

2 Freise A, Strain K. Interferometer techniques for gravitational-wave detection. Living Rev Relativity, 2010, 13: 1-81

3 Hughes S A. Listening to the universe with gravitational-wave astronomy. Ann Phys-New York, 2003, 303: 142-178

4 Amaro-Seoane P, Aoudia S, Babak S, et al. Low-frequency gravitational-wave science with eLISA/NGO. Class Quantum Grav, 2012, 29: 124016

5 Binétruy $\mathrm{P}$, Bohé A, Caprini C, et al. Cosmological backgrounds of gravitational waves and eLISA/NGO: Phase transitions, cosmic strings and other sources. JCAP, 2012, 06: 027

6 Saulson P R. Fundamentals of Interferometric Gravitational Wave Detectors. Singapore: World Scientific, 1994. 12-18

7 Danzmann K, Rüdiger A. LISA technology-concept, status, prospects. Class Quantum Grav, 2003, 20: S1

8 Bender P, Brillet A, Ciufolini I, et al. Lisa pre-phase a report. 2nd ed. Max-Planck-Institut fur Quantenoptik Report No. MPQ 208, Garching, Germany, 1998. 57-61

9 Gerberding O, Sheard B, Bykov I, et al. Phasemeter core for intersatellite laser heterodyne interferometry: modelling, simulations and experiments. Class Quantum Grav, 2013, 30: 235029

10 Heinzel G, Wand V, Garcia A, et al. The LTP interferometer and phasemeter. Class Quantum Grav, 2004, 21: S581

11 Cruise A M, Hoyland D, Aston S M. Implementation of the phasemeter for LISA LTP. Class Quantum Grav, 2005, 22: S165

12 Esteban J J, Bykov I, Marín A F G, et al. Optical ranging and data transfer development for LISA. J Phys: Conf Ser, 2009, 154: 012025

13 Esteban J J, García A F, Eichholz J, et al. Ranging and phase measurement for LISA. J Phys: Conf Ser, 2010, 228: 012045

14 Wand V, Guzmán F, Heinzel G, et al. LISA phasemeter development. AIP Conf Proc, 2006, 873: 689

15 Bykov I, Delgado J J E, Marín A F G, et al. LISA phasemeter development: Advanced prototyping. J Phys: Conf Ser, 2009, 154: 012017

16 Shaddock D A, Ware B, Halverson P, et al. Overview of the LISA Phasemeter. AIP Conf Proc, 2006. 873: 654-660

17 Pollack S E, Jennrich O, Stebbins R T, et al. Status of LISA phase measurement work in the US. Class Quantum Grav, 2003, 20: S193S199

18 Gerberding O, Barke S, Bykov I, et al. Breadboard model of the LISA phasemeter. arXiv preprint, 2012, 1208.6418

19 Gong X, Xu S, Bai S, et al. A scientific case study of an advanced LISA mission. Class Quantum Grav, 2011, 28: 094012

20 Liu H S, Dong Y H, Li Y Q, et al. The evaluation of phasemeter prototype performance for the space gravitational waves detection. Rev Sci Instrum, 2014, 85: 024503

21 Jennrich O, Binetruy P, Colpi M, et al. NGO revealing a hidden universe: Opening a new chapter of discovery. NGO Assessment Study Report, 2011: 84-89

22 McNamara P W. Weak-light phase locking for LISA. Class Quantum Grav, 2005, 22: S243-S247

23 Bender P L. Wavefront distortion and beam pointing for LISA. Class Quantum Grav, 2005, 22: S339-S346

24 Dong Y H, Liu H S, Luo Z R, et al. Methodological demonstration of laser beam pointing control for space gravitational wave detection missions. Rev Sci Instrum, 2014, 85: 074501 